\title{
Longevity in a fish bowl
}

by David Chu, DVM, DACLAM

SCIENTIFIC NAME

Nothobranchius furzeri

TAXONOMY

PHYLUM: Chordata

CLASS: Actinopterygii

ORDER: Cyprinodontiformes

FAMILY: Nothobranchiidae

\section{General description}

African turquoise killifish is a short-lived, teleost fish found in temporary pools of Mozambique and Zimbabwe ${ }^{1}$. To survive the unusually harsh conditions of these pools, N. furzeri spawn daily once they reach sexual maturity $30-40$ days post hatching, and they die within 4-6 months ${ }^{2}$. This is considered the shortest life cycle of any reported captive-bred vertebrate. Adults, reaching a total length of $6.5 \mathrm{~cm}$, exhibit sexual dichromatism and dimorphism, with males displaying predominantly brilliant turquoise scales overlaying crimson skin and females that are smaller, dull and pale brown. Additionally, N. furzeri males have caudal fins with geographically distinctive red and yellow phenotypes ${ }^{1}$.

\section{Husbandry}

African turquoise killifish can be maintained in the laboratory as a brackish warm water species. Although water temperature in the wild can fluctuate as much as $15^{\circ} \mathrm{C}$ (ref. 3 ) and conductivity may range $50-625 \mu \mathrm{S} / \mathrm{cm}$ (ref. 1), it is best to stabilize water parameters in the laboratory at $\mathrm{pH} 6-7.5,23-26^{\circ} \mathrm{C}$ and $3,500-4,500 \mu \mathrm{S} / \mathrm{cm}$. These fish prefer to eat small crustaceans in the wild but will consume typical laboratory fish prey items such as Tubifex worms, Artemia nauplii and Daphnia zooplankton, and captive-reared fish readily accept commercial feed and pellets ${ }^{1}$. It should be noted, however, that Tubifex and other chironomid larvae have been linked to cases of hepatic steatosis ${ }^{4}$. Like other Nothobranchius species, $N$. furzeri is polyandrous and each female can lay 20-50 eggs daily ${ }^{1}$ with male fish displaying a dominance hiearchy ${ }^{3}$. Although wild females deposit eggs directly in pool sediments, captive females readily use sand, peat moss or even zebrafish spawning setups ${ }^{1}$. Like zebrafish, adults consume eggs so one should collect eggs soon

Chu is Staff Veterinarian, Veterinary Service Center at Stanford University, Palo Alto, CA. after oviposition or allow fish to spawn over mesh. Embryos can be incubated on wet substrates or in liquid culture ${ }^{1}$.

\section{Research résumé}

Despite its short life span, the African turquoise killifish completes its life without skipping pathologies and phenotypes associated with old age, which occurs as soon as 2 months after hatching ${ }^{2}$. Multiple studies of its genome $\mathrm{e}^{1,5}$ and RNA transcription ${ }^{6}$ demonstrate that the African turquoise killifish is a viable model to study human aging, and as $N$. furzeri age, they display many stereotypical ailments such as losing body mass, kyphosis, dulling, reduced activity, loss of cognitive functions and organ failures ${ }^{1}$. For oncologists, this species might be of additional interest since African turquoise killifish develop hepatic and renal neoplasia in an age-dependent fashion and at a much higher incidence than other finfish ${ }^{4}$.

Various lines of African killifish allow investigators to study integrative, cellular and even molecular traits of different aging phenoytypes. The grandfather of all inbred lines is the GRZ line, which is highly inbred and with a median lifespan of 3 months, is considered the shortest lived of all killifish ${ }^{1}$. Microinjection techniques allow the creation and germline transmission of stable transgenic lines. For example, the promoter drive for green fluorescent protein has been successfully introduced into N. furzeri ${ }^{7}$. Other techniques are now on the horizon including the CRISPR-Cas9 targeted knock-in approach ${ }^{1,2}$ and ENU random mutagenesis ${ }^{1}$. These powerful tools and this species' short generation time will advance biomedical research, especially in fields of developmental biology and aging.

1. Cellerino, A., Valenzano, D.R. \& Reichard, M. From the bush to the bench: the annual Nothobranchius fishes as a new model system in biology. Biol. Rev. doi:10.111/brv.12183 (published online 28 April 2015).

2. Harel, I. \& Brunet, A. The African turquoise killifish: a model for exploring vertebrate aging and diseases in the fast lane. Cold Spring Harb. Symp. Quant. Biol. doi:10.1101/sqb.2015.80.027524 (published online 7 December 2015).

3. Reichard, M., Polacik, M. \& Sedlacek, 0. Distribution, colour polymorphism and habitat use of the African killifish, Nothobranchius furzeri, the vertebrate with the shortest lifespan. J. Fish Biol. 74, 198-212 (2009).

4. Di Cicco, E., Tozzini, E.T., Rossi, G. \& Cellerino, A. The short-lived annual fish Nothobranchius furzeri shows a typical teleost aging process reinforced by high incidence of age-dependent neoplasia. Exp. Gerontol. 46, 249-256 (2011).

5. Valenzano, D.R. et al. The African turquoise killifish genome provides insights into evolution and genetic architecture of lifespan. Cell 163, 1539-1554 (2015).

6. Petzold, A. et al. The transcript catalogue of the short-lived Nothobranchius furzeri provides insights into age-dependent changes of mRNA levels. BMC Genomics 14, 185 (2013).

7. Hartmann, N. \& Englert, C. A microinjection protocol for the generation of transgenic killifish (Species: Nothobranchius furzeri). Dev. Dyn. 241, 11331141 (2012). 